J. Korean Math. Soc. 51 (2014), No. 2, pp. 325-344

http://dx.doi.org/10.4134/JKMS.2014.51.2.325

\title{
ORE EXTENSIONS OF HOPF GROUP COALGEBRAS
}

\author{
Dingguo Wang and DaOWei Lu
}

\begin{abstract}
The aim of this paper is to generalize the theory of Hopf-Ore extension on Hopf algebras to Hopf group coalgebras. First the concept of Hopf-Ore extension of Hopf group coalgebra is introduced. Then we will give the necessary and sufficient condition for the Ore extensions to become a Hopf group coalgebra, and certain isomorphism between Ore extensions of Hopf group coalgebras are discussed.
\end{abstract}

\section{Introduction}

Ore extensions are main kinds of ring extensions to construct a class of noncommutative rings and algebras. From the point of view of quantum group and Hopf algebra theory, Ore extensions are important for constructing examples of Hopf algebras which are neither commutative nor cocommutative. In recent years, many new examples (often finite dimensional) with special properties were constructed by means of Ore extensions, such as pointed Hopf algebras, co-Frobenius Hopf algebras, and quasitriangular Hopf algebras (see, e.g., $[1,2,5])$.

Panov [6] introduced the concept and equivalent description of a Hopf-Ore extension and given the classifications of Hopf-Ore extensions for some typical Hopf algebras. Multiplier Hopf algebras were introduced by Van Daele [10] as a generalisation of Hopf algebras to the case where the underlying algebra is not necessarily unital. Lihui Zhao and Diming Lu [13] generalized the notion of Ore extension of Hopf Algebras to regular multiplier Hopf algebras and obtained the corresponding result. Hopf group coalgebras were introduced by V. G. Turaev [8, 9]. Hopf group coalgebras generalize usual coalgebras and Hopf algebras, in the sense that we recover these notions in the situation where the group is trivial. Virelizier [11] started an algebraic study of this topic, this was continued by Zunino $[14,15]$ and Wang [12]. It is natural to investigate the Ore extensions of Hopf group coalgebras. This was the motivation of our paper.

The first problem we face is how to define the Ore extensions of Hopf group coalgebras. Here we could resort to the method used in [3] which constructed

Received June 5, 2013.

2010 Mathematics Subject Classification. 16W30, 16 S36.

Key words and phrases. Hopf algebras, Hopf $\pi$-coalgebras, Ore extension. 
the concept of differential calculus on Hopf group coalgebras, that is, let $A=$ $\left\{A_{\alpha}\right\}_{\alpha \in \pi}$ be a Hopf group coalgebra, and $R=\left\{R_{\alpha}=A_{\alpha}\left[y_{\alpha} ; \sigma_{\alpha}, \delta_{\alpha}\right]\right\}_{\alpha \in \pi}$, where for any $\alpha \in \pi, A_{\alpha}\left[y_{\alpha} ; \sigma_{\alpha}, \delta_{\alpha}\right]$ is the Ore extension of $A_{\alpha}$. Then we make $R=\left\{R_{\alpha}\right\}_{\alpha \in \pi}$ a Hopf group coalgebra. The comultiplication and counit could be extended from $A$ to $R$ naturally.

In Section 2, we recall the definition of Hopf group coalgebras and some basic facts about Hopf group coalgebras. In Section 3, we first introduce the notion of an Ore extension for a Hopf group coalgebra, and extend $\Delta$ and $\varepsilon$ from $A$ to $R$ such that $R=\left\{R_{\alpha}\right\}_{\alpha \in \pi}$ becomes a Hopf group coalgebra. In the main theorem of this article, we give a necessary and sufficient condition for Ore extensions of a Hopf group coalgebra to be a Hopf group coalgebra. In Section 4, we will consider the isomorphisms between Ore extensions for different Hopf group coalgebras and give the sufficient conditions.

\section{Hopf group coalgebra}

For convenience of the reader we recall the standard definitions of Hopf algebras, see for instance $[4,7]$. Throughout this paper, we let $\pi$ be a discrete group(with neutral element 1) and $k$ be a field. All algebras are supposed to be over $k$. If $U$ and $V$ are $k$-space, $\tau_{U, V}: U \otimes V \longrightarrow V \otimes U$ will denote the flip defined by $\tau_{U, V}(u \otimes v)=v \otimes u$.

Definition 2.1. A $\pi$-coalgebra(over $k$ ) is a family $C=\left\{C_{\alpha}\right\}_{\alpha \in \pi}$ of $k$-spaces endowed with a family $k$-linear maps (the comultiplication) and a $k$-linear map $\varepsilon: C_{1} \longrightarrow k$ (the counit) such that

(a) $\Delta$ is a coassociative in the sense that, for any $\alpha, \beta, \gamma \in \pi$,

$$
\left(\Delta_{\alpha, \beta} \otimes i d_{C_{\gamma}}\right) \Delta_{\alpha \beta, \gamma}=\left(i d_{C_{\alpha}} \otimes \Delta_{\beta, \gamma}\right) \Delta_{\alpha, \beta \gamma}
$$

(b) for all $\alpha \in \pi$,

$$
\left(i d_{C_{\alpha}} \otimes \varepsilon\right) \Delta_{\alpha, 1}=i d_{C_{\alpha}}=\left(\varepsilon \otimes i d_{C_{\alpha}}\right) \Delta_{1, \alpha} .
$$

Note that $\left(C_{1}, \Delta_{1,1}, \varepsilon\right)$ is a coalgebra in the usual sense.

Sweedler's notation. We extend the Sweedler notation in the following way: for any $\alpha, \beta \in \pi$ and $c \in C_{\alpha \beta}$, we write

$$
\Delta_{\alpha, \beta}(c)=\sum_{(c)} c_{(1, \alpha)} \otimes c_{(2, \beta)} \in C_{\alpha} \otimes C_{\beta} .
$$

Or shortly, if we leave the summation implicitly, $\Delta_{\alpha, \beta}(c)=c_{(1, \alpha)} \otimes c_{(2, \beta)}$.

The coassociativity axiom gives that, for any $\alpha, \beta, \gamma \in \pi$ and $c \in C_{\alpha \beta \gamma}$,

$$
c_{(1, \alpha \beta)(1, \alpha)} \otimes c_{(1, \alpha \beta)(2, \beta)} \otimes c_{(2, \gamma)}=c_{(1, \alpha)} \otimes c_{(2, \beta \gamma)(1, \beta)} \otimes c_{(2, \beta \gamma)(2, \gamma)} .
$$

This element of $C_{\alpha} \otimes C_{\beta} \otimes C_{\gamma}$ is written as $c_{(1, \alpha)} \otimes c_{(2, \beta} \otimes c_{(3, \gamma)}$. For any $c \in$ $C_{\alpha_{1} \cdots \alpha_{n}}$, by iterating the procedure we define inductively $c_{\left(1, \alpha_{1}\right)} \otimes \cdots \otimes c_{\left(n, \alpha_{n}\right)}$. 
Definition 2.2. Let $C=\left(\left\{C_{\alpha}, \Delta, \varepsilon\right\}\right)$ be a $\pi$-coalgebra and $A$ be an algebra with multiplication $m$ and unit element $1_{A}$. For any $f \in \operatorname{Hom}_{k}\left(C_{\alpha}, A\right)$ and $g \in \operatorname{Hom}_{k}\left(C_{\beta}, A\right)$, we define their convolution product by

$$
f * g=m(f \otimes g) \Delta_{\alpha, \beta} \in \operatorname{Hom}_{k}\left(C_{\alpha \beta}, A\right) .
$$

Using (2.1), one verifies that the $k$-space

$$
\operatorname{Conv}(C, A)=\oplus_{\alpha \in \pi} \operatorname{Hom}_{k}\left(C_{\alpha}, A\right),
$$

endowed with the convolution product $*$ and the unit element $\varepsilon 1_{A}$, is a $\pi$-graded algebra, called convolution algebra.

Definition 2.3. A Hopf $\pi$-coalgebra is a $\pi$-coalgebra $H=\left(\left\{H_{\alpha}, \Delta, \varepsilon\right\}\right)$ endowed with a family $S=\left\{S_{\alpha}: \quad H_{\alpha} \longrightarrow H_{\alpha^{-1}}\right\}_{\alpha \in \pi}$ of $k$-linear maps (the antipode) such that

(a) each $H_{\alpha}$ is an algebra with multiplication $m_{\alpha}$ and $1_{\alpha} \in H_{\alpha}$;

(b) $\varepsilon: H_{1} \longrightarrow k$ and $\Delta_{\alpha, \beta}: H_{\alpha \beta} \longrightarrow H_{\alpha} \otimes H_{\beta}$ (for all $\alpha, \beta \in \pi$ ) are algebra homomorphisms;

(c) for any $\alpha \in \pi$,

$m_{\alpha}\left(S_{\alpha^{-1}} \otimes i d_{H_{\alpha}}\right) \Delta_{\alpha_{-1}, \alpha}=\varepsilon 1_{\alpha}=m_{\alpha}\left(i d_{H_{\alpha}} \otimes S_{\alpha^{-1}}\right) \Delta_{\alpha, \alpha^{-1}}: H_{1} \rightarrow H_{\alpha}$.

Note that $\left(H_{1}, m_{1}, 1_{1}, \Delta_{1,1}, \varepsilon, S_{1}\right)$ is a Hopf algebra in the usual sense of the word. We call it the neutral component of $H$. And axiom $(c)$ says that $S_{\alpha}$ is the inverse of $i d_{H_{\alpha}}$ in the convolution algebra $\operatorname{Conv}\left(H_{1}, H_{\alpha}\right)$.

Lemma 2.4. Let $H=\left(\left\{H_{\alpha}\right\}_{\alpha \in \pi}, \Delta, \varepsilon, S\right)$ be a Hopf $\pi$-coalgebra. Then

(a) $\Delta_{\beta^{-1}, \alpha^{-1}} S_{\alpha \beta}=\tau_{H_{\alpha^{-1}}, H_{\beta^{-1}}}\left(S_{\alpha} \otimes S_{\beta}\right) \Delta_{\alpha, \beta}$ for any $\alpha, \beta \in \pi$,

(b) $\varepsilon S_{1}=\varepsilon$,

(c) $S_{\alpha}(a b)=S_{\alpha}(b) S_{\beta}(a)$ for any $\alpha \in \pi$ and $a, b \in H_{\alpha}$,

(d) $S_{\alpha}\left(1_{\alpha}\right)=1_{\alpha^{-1}}$ for any $\alpha \in \pi$.

\section{Main theorem}

In this section, we will define the Ore extension of a Hopf group coalgebra and prove the criterion for an Ore extension of a Hopf group coalgebra to be a Hopf group coalgebra.

Definition 3.1. Let $A$ be a $k$-algebra. Consider an endomorphism $\sigma$ of the algebra $A$ over $k$ and a $\sigma$-derivation $\delta$ of $A$. This means that

$$
\delta(a b)=\sigma(a) \delta(b)+\delta(a) b .
$$

The Ore extension $R=A[y ; \sigma, \delta]$ of the $k$-algebra $R$ generated by the variable $y$ and the algebra $A$ with the relation

$$
y a=\sigma(a) y+\delta(a)
$$

for any $a \in A$.

Now with the definition of Hopf-Ore extension [6], we can define the Hopf group coalgebra Ore extension. 
Definition 3.2. Let $A=\left\{A_{\alpha}\right\}_{\alpha \in \pi}$ be a Hopf group coalgebra, the family $R=\left\{R_{\alpha}=A_{\alpha}\left[y_{\alpha} ; \sigma_{\alpha}, \delta_{\alpha}\right]\right\}_{\alpha \in \pi}$ of $k$-spaces is called the Hopf Group coalgebra Ore extension if $R=\left\{R_{\alpha}\right\}_{\alpha \in \pi}$ is also a Hopf group coalgebra, where for any $\alpha \in \pi, A_{\alpha}\left[y_{\alpha} ; \sigma_{\alpha}, \delta_{\alpha}\right]$ is the Ore extension of $A_{\alpha}$, and there exist $r_{\alpha}^{1}, r_{\alpha}^{2} \in A_{\alpha}$ such that

and

$$
\Delta_{\alpha, \beta}\left(y_{\alpha \beta}\right)=y_{\alpha} \otimes r_{\beta}^{2}+r_{\alpha}^{1} \otimes y_{\beta},
$$

$$
\Delta_{\beta, \alpha}\left(y_{\beta \alpha}\right)=y_{\beta} \otimes r_{\alpha}^{2}+r_{\beta}^{1} \otimes y_{\alpha} .
$$

Note that $R_{1}=A_{1}\left[y_{1} ; \sigma_{1}, \delta_{1}\right]$ is the Hopf-Ore extension in the sense of [6].

Now that $R=\left\{R_{\alpha}\right\}_{\alpha \in \pi}$ is a Hopf group coalgebra, if we apply the axioms of (2.1) to Definition 3.2, we have on one hand

$$
\begin{aligned}
\left(\Delta_{\alpha, \beta} \otimes i d_{R_{\gamma}}\right) \Delta_{\alpha \beta, \gamma}\left(y_{\alpha \beta \gamma}\right) & =\left(\Delta_{\alpha, \beta} \otimes i d_{R_{\gamma}}\right)\left(y_{\alpha \beta} \otimes r_{\gamma}^{2}+r_{\alpha \beta}^{1} \otimes y_{\gamma}\right) \\
& =\Delta_{\alpha, \beta}\left(y_{\alpha \beta}\right) \otimes r_{\gamma}^{2}+\Delta_{\alpha, \beta}\left(r_{\alpha \beta}^{1}\right) \otimes y_{\gamma} \\
& =\left(y_{\alpha} \otimes r_{\beta}^{2}+r_{\alpha}^{1} \otimes y_{\beta}\right) \otimes r_{\gamma}^{2}+\Delta_{\alpha, \beta}\left(r_{\alpha \beta}^{1}\right) \otimes y_{\gamma} \\
& =y_{\alpha} \otimes r_{\beta}^{2} \otimes r_{\gamma}^{2}+r_{\alpha}^{1} \otimes y_{\beta} \otimes r_{\gamma}^{2}+\Delta_{\alpha, \beta}\left(r_{\alpha \beta}^{1}\right) \otimes y_{\gamma} .
\end{aligned}
$$

On the other hand

$$
\begin{aligned}
\left(i d_{R_{\alpha}} \otimes \Delta_{\beta, \gamma}\right) \Delta_{\alpha, \beta \gamma}\left(y_{\alpha \beta \gamma}\right) & =\left(i d_{R_{\alpha}} \otimes \Delta_{\beta, \gamma}\right)\left(y_{\alpha} \otimes r_{\beta \gamma}^{2}+r_{\alpha}^{1} \otimes y_{\beta \gamma}\right) \\
& =y_{\alpha} \otimes \Delta_{\beta, \gamma}\left(r_{\beta \gamma}^{2}\right)+r_{\alpha}^{1} \otimes\left(y_{\beta} \otimes r_{\gamma}^{2}+r_{\beta}^{1} \otimes y_{\gamma}\right) \\
& =y_{\alpha} \otimes \Delta_{\beta, \gamma}\left(r_{\beta \gamma}^{2}\right)+r_{\alpha}^{1} \otimes y_{\beta} \otimes r_{\gamma}^{2}+r_{\alpha}^{1} \otimes r_{\beta}^{1} \otimes y_{\gamma} .
\end{aligned}
$$

We obtain

$$
\Delta_{\alpha, \beta}\left(r_{\alpha \beta}^{1}\right)=r_{\alpha}^{1} \otimes r_{\beta}^{1}, \Delta_{\alpha, \beta}\left(r_{\alpha \beta}^{2}\right)=r_{\alpha}^{2} \otimes r_{\beta}^{2}
$$

for any $\alpha, \beta, \gamma \in \pi$.

Lemma 3.3. If $R=\left\{R_{\alpha}\right\}_{\alpha \in \pi}$ is the Hopf group coalgebra Ore extension of the Hopf group coalgebra $A=\left\{A_{\alpha}\right\}_{\alpha \in \pi}$ as above, then for any $\alpha, \beta \in \pi$

(a) $r_{\alpha}^{1}$ and $r_{\alpha}^{2}$ are invertible in $A_{\alpha}$,

(b) the equalities:

$$
\Delta_{\alpha, \beta}\left(\left(r_{\alpha \beta}^{1}\right)^{-1}\right)=\left(r_{\alpha}^{1}\right)^{-1} \otimes\left(r_{\beta}^{1}\right)^{-1},
$$

and

$$
\Delta_{\alpha, \beta}\left(\left(r_{\alpha \beta}^{2}\right)^{-1}\right)=\left(r_{\alpha}^{2}\right)^{-1} \otimes\left(r_{\beta}^{2}\right)^{-1} .
$$

Proof. (1) First of all, from (3.3) we can see that $r_{1}^{1}$ is a group-like element in $A_{1}$, so $\varepsilon\left(r_{1}^{1}\right)=1$.

$$
\begin{aligned}
m_{\alpha}\left(S_{\alpha^{-1}} \otimes i d\right) \Delta_{\alpha^{-1}, \alpha}\left(r_{1}^{1}\right) & =m_{\alpha}\left(S_{\alpha^{-1}} \otimes i d\right)\left(r_{\alpha^{-1}}^{1} \otimes r_{\alpha}^{1}\right) \\
& =S_{\alpha^{-1}}\left(r_{\alpha^{-1}}^{1}\right) r_{\alpha}^{1} \\
& =\varepsilon\left(r_{1}^{1}\right) 1_{\alpha} \\
& =1_{\alpha} .
\end{aligned}
$$


So we have $S_{\alpha^{-1}}\left(r_{\alpha^{-1}}^{1}\right)=\left(r_{\alpha}^{1}\right)^{-1}$, and similarly we get $S_{\alpha^{-1}}\left(r_{\alpha^{-1}}^{2}\right)=\left(r_{\alpha}^{2}\right)^{-1}$ for any $\alpha \in \pi$.

(2) By Lemma 2.4, We have

$$
\begin{aligned}
\Delta_{\alpha, \beta}\left(\left(r_{\alpha \beta}^{1}\right)^{-1}\right) & =\Delta_{\alpha, \beta}\left(S_{(\alpha \beta)^{-1}}\left(r_{(\alpha \beta)^{-1}}^{1}\right)\right) \\
& =\Delta_{\alpha, \beta}\left(S_{\beta^{-1} \alpha^{-1}}\left(r_{\beta^{-1} \alpha^{-1}}^{1}\right)\right) \\
& =\tau\left(S_{\beta^{-1}} \otimes S_{\alpha^{-1}}\right)\left(\Delta_{\beta^{-1} \alpha^{-1}}\left(r_{\beta^{-1} \alpha^{-1}}^{1}\right)\right) \\
& =\tau\left(S_{\beta^{-1}}\left(r_{\beta^{-1}}^{1}\right) \otimes S_{\alpha^{-1}}\left(r_{\alpha^{-1}}^{1}\right)\right) \\
& =\left(r_{\alpha}^{1}\right)^{-1} \otimes\left(r_{\beta}^{1}\right)^{-1}
\end{aligned}
$$

Similarly we can prove the other equality.

Replacing the generating elements $y_{\alpha}$ by $y_{\alpha}^{\prime}=y_{\alpha}\left(r_{\alpha}^{2}\right)^{-1}$ and $r_{\alpha}^{1}\left(r_{\alpha}^{2}\right)^{-1}$ by $r_{\alpha}$, we see that

$$
\begin{aligned}
\Delta_{\alpha, \beta}\left(y_{\alpha \beta}^{\prime}\right) & =\Delta_{\alpha, \beta}\left(y_{\alpha \beta}\left(r_{\alpha \beta}^{2}\right)^{-1}\right) \\
& =\Delta_{\alpha, \beta}\left(y_{\alpha \beta}\right) \cdot \Delta_{\alpha, \beta}\left(\left(r_{\alpha \beta}^{2}\right)^{-1}\right) \\
& =\left(y_{\alpha} \otimes r_{\beta}^{2}+r_{\alpha}^{1} \otimes y_{\beta}\right) \cdot\left(\left(r_{\alpha}^{2}\right)^{-1} \otimes\left(r_{\beta}^{2}\right)^{-1}\right) \\
& =y_{\alpha}\left(r_{\alpha}^{2}\right)^{-1} \otimes 1+r_{\alpha}^{1}\left(r_{\alpha}^{2}\right)^{-1} \otimes y_{\beta}\left(r_{\beta}^{2}\right)^{-1} \\
& =y_{\alpha}^{\prime} \otimes 1+r_{\alpha} \otimes y_{\beta}^{\prime} .
\end{aligned}
$$

And

$$
\begin{aligned}
\Delta_{\alpha, \beta}\left(r_{\alpha \beta}\right) & =\Delta_{\alpha, \beta}\left(r_{\alpha \beta}^{1}\left(r_{\alpha \beta}^{2}\right)^{-1}\right) \\
& =\Delta_{\alpha, \beta}\left(r_{\alpha \beta}^{1}\right) \cdot \Delta_{\alpha, \beta}\left(\left(r_{\alpha \beta}^{2}\right)^{-1}\right) \\
& =\left(r_{\alpha}^{1} \otimes r_{\beta}^{1}\right) \cdot\left(\left(r_{\alpha}^{2}\right)^{-1} \otimes\left(r_{\beta}^{2}\right)^{-1}\right) \\
& =r_{\alpha}^{1}\left(r_{\alpha}^{2}\right)^{-1} \otimes r_{\beta}^{1}\left(r_{\beta}^{2}\right)^{-1} \\
& =r_{\alpha} \otimes r_{\beta} .
\end{aligned}
$$

Preserving the above notations, we assume in what follows that the elements $\left\{y_{\alpha}\right\}_{\alpha \in \pi}$ in the Hopf group coalgebra-Ore extension satisfying the relations

$$
\Delta_{\alpha, \beta}\left(y_{\alpha \beta}\right)=y_{\alpha} \otimes 1+r_{\alpha} \otimes y_{\beta}
$$

for some elements $r_{\alpha} \in A_{\alpha}$ satisfying $\Delta_{\alpha, \beta}\left(r_{\alpha \beta}\right)=r_{\alpha} \otimes r_{\beta}$.

As usual, $A d_{r_{\alpha}}(a)=r_{\alpha} a S_{\alpha^{-1}}\left(r_{\alpha}^{-1}\right)=r_{\alpha} a\left(r_{\alpha}\right)^{-1}$.

Lemma 3.4. If $R=\left\{A_{\alpha}\left[y_{\alpha} ; \sigma_{\alpha}, \delta_{\alpha}\right]\right\}_{\alpha \in \pi}$ is a Hopf group coalgebra-Ore extension of the Hopf group coalgebra $A=\left\{A_{\alpha}\right\}_{\alpha \in \pi}$, then

$$
S_{\alpha^{-1}}\left(y_{\alpha^{-1}}\right)=-\left(r_{\alpha}\right)^{-1} y_{\alpha}
$$

where $\left(r_{\alpha}\right)^{-1}=S_{\alpha^{-1}}\left(r_{\alpha^{-1}}\right)$.

Proof. $\left(r_{\alpha}\right)^{-1}=S_{\alpha^{-1}}\left(r_{\alpha^{-1}}\right)$ is easy to check. Now we have

$$
m_{\alpha}\left(S_{\alpha^{-1}} \otimes i d_{A_{\alpha}}\right) \Delta_{\alpha^{-1}, \alpha}\left(y_{1}\right)=\varepsilon\left(y_{1}\right) 1_{\alpha}=0,
$$


by $(3.7)$, so

$$
S_{\alpha^{-1}}\left(y_{\alpha^{-1}}\right)+S_{\alpha^{-1}}\left(r_{\alpha^{-1}}\right) y_{\alpha}=0
$$

then

$$
S_{\alpha^{-1}}\left(y_{\alpha^{-1}}\right)=-S_{\alpha^{-1}}\left(r_{\alpha^{-1}}\right) y_{\alpha}=-\left(r_{\alpha}\right)^{-1} y_{\alpha} .
$$

Now we will give the main result.

Theorem 3.5. The Ore extension $R=\left\{A_{\alpha}\left[y_{\alpha} ; \sigma_{\alpha}, \delta_{\alpha}\right]\right\}_{\alpha \in \pi}$ of the Hopf group coalgebra $A=\left\{A_{\alpha}\right\}_{\alpha \in \pi}$ is a Hopf group coalgebra-Ore extension if and only if there exists a group-like element $r=\left\{r_{\alpha}\right\}_{\alpha \in \pi}$ such that the following conditions hold:

(a) there is a character $\chi: A_{1} \longrightarrow k$ such that for any $\alpha \in \pi$

$$
\sigma_{\alpha}(a)=\sum \chi\left(a_{(1,1)}\right) a_{(2, \alpha)},
$$

where $a \in A_{\alpha}$

(b) the following relation holds:

$$
\sum \chi\left(a_{(1,1)}\right) a_{(2, \alpha)}=A d_{r_{\alpha}}\left(a_{(1, \alpha)}\right) \chi\left(a_{(2,1)}\right),
$$

(c) the $\sigma_{\alpha}$-derivation $\delta$ satisfies the relation

$$
\Delta_{\alpha, \beta} \delta_{\alpha \beta}(a)=\sum \delta_{\alpha}\left(a_{(1, \alpha)}\right) \otimes a_{(2, \beta)}+r_{\alpha} a_{(1, \alpha)} \otimes \delta_{\beta}\left(a_{(2, \beta)}\right) .
$$

Proof. The proof is presented under three headings. At step 1 we show that the comultiplication $\Delta=\left\{\Delta_{\alpha}\right\}_{\alpha \in \pi}$ can be extended to $R=\left\{A_{\alpha}\left[y_{\alpha} ; \sigma_{\alpha}, \delta_{\alpha}\right]\right\}_{\alpha \in \pi}$ by (3.7) if and only if relations (3.9)-(3.11) hold. At step 2 we prove that $R_{1}$ admits an extension of the counit from $A_{1}$ (in fact this has been proved in [6]). At step 3 we show that $R$ has antipode $S$ extending the antipode $\left.S\right|_{A}$ by (3.8).

Step 1. Comultiplication. Assume that the comultiplication $\left.\Delta\right|_{A}$ can be extended to $R=\left\{A_{\alpha}\left[y_{\alpha} ; \sigma_{\alpha}, \delta_{\alpha}\right]\right\}_{\alpha \in \pi}$ by (3.7). Then the homomorphism $\Delta$ preserve the relation

$$
y_{\alpha} a=\sigma_{\alpha}(a) y_{\alpha}+\delta_{\alpha}(a)
$$

for any $\alpha \in \pi$ and $a \in A_{\alpha}$, i.e.,

$$
\Delta_{\alpha, \beta}\left(y_{\alpha \beta}\right) \Delta_{\alpha, \beta}(a)=\Delta_{\alpha, \beta} \sigma_{\alpha \beta}(a) \Delta_{\alpha, \beta}\left(y_{\alpha \beta}\right)+\Delta_{\alpha, \beta} \delta_{\alpha \beta}(a)
$$

for any $a \in A_{\alpha \beta}$. We have

$$
\begin{aligned}
& \Delta_{\alpha, \beta}\left(y_{\alpha \beta}\right) \Delta_{\alpha, \beta}(a) \\
= & \sum_{(a)}\left(y_{\alpha} \otimes 1+r_{\alpha} \otimes y_{\beta}\right)\left(a_{(1, \alpha)} \otimes a_{(2, \beta)}\right) \\
= & \sum_{(a)} y_{\alpha} a_{(1, \alpha)} \otimes a_{(2, \beta)}+r_{\alpha} a_{(1, \alpha)} \otimes y_{\beta} a_{(2, \beta)} \\
= & \sum_{(a)} \sigma_{\alpha}\left(a_{(1, \alpha)}\right) y_{\alpha} \otimes a_{(2, \beta)}+\delta_{\alpha}\left(a_{(1, \alpha)}\right) \otimes a_{(2, \beta)} \\
& +r_{\alpha} a_{(1, \alpha)} \otimes \sigma_{\beta}\left(a_{(2, \beta)}\right) y_{\beta}+r_{\alpha} a_{(1, \alpha)} \otimes \delta_{\beta}\left(a_{(2, \beta)}\right)
\end{aligned}
$$




$$
\begin{aligned}
= & \sum_{(a)}\left(\sigma_{\alpha}\left(a_{(1, \alpha)}\right) \otimes a_{(2, \beta)}\right)\left(y_{\alpha} \otimes 1\right)+r_{\alpha} a_{(1, \alpha)} \otimes \sigma_{\beta}\left(a_{(2, \beta)}\right) y_{\beta} \\
& +\delta_{\alpha}\left(a_{(1, \alpha)}\right) \otimes a_{(2, \beta)}+r_{\alpha} a_{(1, \alpha)} \otimes \delta_{\beta}\left(a_{(2, \beta)}\right) \\
= & \sum_{(a)}\left(\sigma_{\alpha}\left(a_{(1, \alpha)}\right) \otimes a_{(2, \beta)}\right)\left(y_{\alpha} \otimes 1\right)+\left(r_{\alpha} a_{(1, \alpha)} r_{\alpha}^{-1} \otimes \sigma_{\beta}\left(a_{(2, \beta)}\right)\left(r_{\alpha} \otimes y_{\beta}\right)\right. \\
& +\delta_{\alpha}\left(a_{(1, \alpha)}\right) \otimes a_{(2, \beta)}+r_{\alpha} a_{(1, \alpha)} \otimes \delta_{\beta}\left(a_{(2, \beta)}\right) .
\end{aligned}
$$

And

$$
\begin{aligned}
& \Delta_{\alpha, \beta} \sigma_{\alpha \beta}(a) \Delta_{\alpha, \beta}\left(y_{\alpha \beta}\right)+\Delta_{\alpha, \beta} \delta_{\alpha \beta}(a) \\
= & \Delta_{\alpha, \beta} \sigma_{\alpha \beta}(a)\left(y_{\alpha} \otimes 1+r_{\alpha} \otimes y_{\beta}\right)+\Delta_{\alpha, \beta} \delta_{\alpha \beta}(a) \\
= & \Delta_{\alpha, \beta} \sigma_{\alpha \beta}(a)\left(y_{\alpha} \otimes 1\right)+\Delta_{\alpha, \beta} \sigma_{\alpha \beta}(a)\left(r_{\alpha} \otimes y_{\beta}\right)+\Delta_{\alpha, \beta} \delta_{\alpha \beta}(a) .
\end{aligned}
$$

It is clear that $\Delta$ preserves (3.12) if and only if the following relations hold:

$$
\begin{gathered}
\Delta_{\alpha, \beta} \sigma_{\alpha \beta}(a)=\sum_{(a)} A d_{r_{\alpha}}\left(a_{(1, \alpha)}\right) \otimes \sigma_{\beta}\left(a_{(2, \beta)}\right), \\
\Delta_{\alpha, \beta} \delta_{\alpha \beta}(a)=\sum_{(a)} \delta_{\alpha}\left(a_{(1, \alpha)}\right) \otimes a_{(2, \beta)}+r_{\alpha} a_{(1, \alpha)} \otimes \delta_{\beta}\left(a_{(2, \beta)}\right)
\end{gathered}
$$

for any $a \in A_{\alpha \beta}$. The last equation coincides with (3.11).

Let us show that (3.14) and (3.15) imply (3.10) and (3.11). Define a family of maps $\left\{\chi_{\alpha}: A_{1} \longrightarrow A_{\alpha}\right\}_{\alpha \in \pi}$ by

$$
\chi_{\alpha}(a)=\sum_{(a)} \sigma_{\alpha}\left(a_{(1, \alpha)}\right) S_{\alpha^{-1}}\left(a_{\left(2, \alpha^{-1}\right)}\right)
$$

for any $a \in A_{1}$.

Obvious compulation gives

$$
\begin{aligned}
& \Delta_{\alpha, 1}\left(\chi_{\alpha}(a)\right) \\
= & \sum_{(a)} \Delta_{\alpha, 1}\left(\sigma_{\alpha}\left(a_{(1, \alpha)}\right) S_{\alpha^{-1}}\left(a_{\left(2, \alpha^{-1}\right)}\right)\right) \\
= & \sum_{(a)} \Delta_{\alpha, 1}\left(\sigma_{\alpha}\left(a_{(1, \alpha)}\right)\right) \Delta_{\alpha, 1} S_{\alpha^{-1}}\left(a_{\left(2, \alpha^{-1}\right)}\right) \\
= & \sum_{(a)}\left[\sigma_{\alpha}\left(a_{(1, \alpha)(1, \alpha)}\right) \otimes a_{(1, \alpha)(2,1)}\right]\left[S_{\alpha^{-1}}\left(a_{\left(2, \alpha^{-1}\right)\left(2, \alpha^{-1}\right)}\right) \otimes S_{1}\left(a_{\left(2, \alpha^{-1}\right)(1,1)}\right)\right] \\
= & \sum_{(a)} \sigma_{\alpha}\left(a_{(1, \alpha)(1, \alpha)}\right) S_{\alpha^{-1}}\left(a_{\left(2, \alpha^{-1}\right)\left(2, \alpha^{-1}\right)}\right) \otimes a_{(1, \alpha)(2,1)} S_{1}\left(a_{\left(2, \alpha^{-1}\right)(1,1)}\right) \\
= & \sum_{(a)} \sigma_{\alpha}\left(a_{(1, \alpha)}\right) S_{\alpha^{-1}}\left(a_{\left(4, \alpha^{-1}\right)}\right) \otimes a_{(2,1)} S_{1}\left(a_{(3,1)}\right)
\end{aligned}
$$


$=\chi_{\alpha}(a) \otimes 1$,

and

$$
\begin{aligned}
& \Delta_{1, \alpha}\left(\chi_{\alpha}(a)\right) \\
= & \sum_{(a)} \Delta_{1, \alpha}\left(\sigma_{\alpha}\left(a_{(1, \alpha)}\right) \Delta_{1, \alpha} S_{\alpha^{-1}}\left(a_{\left(2, \alpha^{-1}\right)}\right)\right. \\
= & \sum_{(a)}\left[\sigma_{1}\left(a_{(1, \alpha)(1,1)}\right) \otimes a_{(1, \alpha)(2, \alpha)}\right]\left[\tau\left(S_{\alpha^{-1}} \otimes S_{1}\right) \Delta_{\alpha^{-1}, 1}\left(a_{\left(2, \alpha^{-1}\right)}\right)\right] \\
= & \sum_{(a)}\left[\sigma_{1}\left(a_{(1, \alpha)(1,1)}\right) \otimes a_{(1, \alpha)(2, \alpha)}\right]\left[S_{1}\left(a_{\left(2, \alpha^{-1}\right)(2,1)}\right) \otimes S_{\alpha^{-1}}\left(a_{\left(2, \alpha^{-1}\right)\left(1, \alpha^{-1}\right)}\right)\right] \\
= & \sum_{(a)} \sigma_{1}\left(a_{(1, \alpha)(1,1)}\right) S_{1}\left(a_{\left(2, \alpha^{-1}\right)(2,1)}\right) \otimes a_{(1, \alpha)(2, \alpha)} S_{\alpha^{-1}}\left(a_{\left(2, \alpha^{-1}\right)\left(1, \alpha^{-1}\right)}\right) \\
= & \sum_{(a)} \sigma_{1}\left(a_{(1,1)}\right) S_{1}\left(a_{(4,1)}\right) \otimes a_{(2, \alpha)} S_{\alpha^{-1}}\left(a_{\left(3, \alpha^{-1}\right)}\right) \\
= & \sum_{(a)} \sigma_{1}\left(a_{(1,1)}\right) S_{1}\left(a_{(2,1)}\right) \otimes 1_{\alpha} \\
= & \chi_{1}(a) \otimes 1_{\alpha},
\end{aligned}
$$

so we have

$$
\begin{aligned}
\chi_{\alpha}(a) & =(\varepsilon \otimes i d) \Delta_{1, \alpha}\left(\chi_{\alpha}(a)\right)=\sum_{(a)} \varepsilon\left(\sigma_{1}\left(a_{(1,1)}\right) S_{1}\left(a_{(2,1)}\right)\right) 1_{\alpha} \\
& =\sum_{(a)} \varepsilon\left(\sigma_{1}\left(a_{(1,1)}\right) \varepsilon\left(S_{1}\left(a_{(2,1)}\right)\right) 1_{\alpha}=\sum_{(a)} \varepsilon\left(\sigma_{1}\left(a_{(1,1)}\right) \varepsilon\left(a_{(2,1)}\right) 1_{\alpha}\right.\right. \\
& =\varepsilon\left(\sigma_{1}(a)\right) 1_{\alpha} .
\end{aligned}
$$

Also we have for any $\alpha \in \pi, \chi_{\alpha}(a)=\varepsilon\left(\chi_{1}(a)\right) 1_{\alpha}$. Indeed from Theorem 1.3 of [6], we know that for any $a \in A_{1}, \chi_{1}(a)$ belongs to $k$, thus $\chi_{\alpha}(a)$ could be identified with an element in $k$ as well. One can regard $\chi_{\alpha}$ as a mapping $\chi: A_{1} \longrightarrow k$. Since for any $\alpha \in \pi, \sigma_{\alpha}$ is an endomorphism, it follows that

$$
\begin{aligned}
\chi_{\alpha}(a b) & =\sum_{(a)(b)} \sigma_{\alpha}\left(a_{(1, \alpha)} b_{(1, \alpha)}\right) S_{\alpha^{-1}}\left(a_{\left(2, \alpha^{-1}\right)} b_{\left(2, \alpha^{-1}\right)}\right) \\
& =\sum_{(a)(b)} \sigma_{\alpha}\left(a_{(1, \alpha)}\right) \sigma_{\alpha}\left(b_{(1, \alpha)}\right) S_{\alpha^{-1}}\left(b_{\left(2, \alpha^{-1}\right)}\right) S_{\alpha^{-1}}\left(a_{\left(2, \alpha^{-1}\right)}\right) \\
& =\sum_{(a)} \sigma_{\alpha}\left(a_{(1, \alpha)}\right) \chi_{\alpha}(b) S_{\alpha^{-1}}\left(a_{\left(2, \alpha^{-1}\right)}\right) \\
& =\chi_{\alpha}(a) \chi_{\alpha}(b)
\end{aligned}
$$




$$
\begin{aligned}
\chi_{\alpha}(a+b) & =\sum_{(a)(b)} \sigma_{\alpha}\left((a+b)_{(1, \alpha)}\right) S_{\alpha^{-1}}\left((a+b)_{\left(2, \alpha^{-1}\right)}\right) \\
& =\sum_{(a)(b)} \sigma_{\alpha}\left(a_{(1, \alpha)}\right) S_{\alpha^{-1}}\left(a_{\left(2, \alpha^{-1}\right)}\right)+\sigma_{\alpha}\left(b_{(1, \alpha)}\right) S_{\alpha^{-1}}\left(b_{\left(2, \alpha^{-1}\right)}\right) \\
& =\chi_{\alpha}(a)+\chi_{\alpha}(b)
\end{aligned}
$$

One can recover $\sigma_{\alpha}$ from $\chi_{\alpha}(a)$. In fact for any $a \in A_{\alpha}, \Delta_{1, \alpha}(a)=\sum_{(a)} a_{(1,1)} \otimes$ $a_{(2, \alpha)}$ and

$$
\begin{aligned}
\sum_{(a)} \chi_{\alpha}\left(a_{(1,1)}\right) a_{(2, \alpha)} & =\sum_{(a)} \sigma_{\alpha}\left(a_{(1,1)(1, \alpha)}\right) S_{\alpha^{-1}}\left(a_{(1,1)\left(2, \alpha^{-1}\right)}\right) a_{(2, \alpha)} \\
& =\sum_{(a)} \sigma_{\alpha}\left(a_{(1, \alpha)}\right) S_{\alpha^{-1}}\left(a_{\left(2, \alpha^{-1}\right)}\right) a_{(3, \alpha)} \\
& =\sigma_{\alpha}(a)
\end{aligned}
$$

This proves (3.9).

Substituting $\sigma_{\alpha}$ into (3.15), we obtain

$$
\begin{aligned}
\sum_{(a)} \Delta_{1, \alpha}\left(\chi_{\alpha}\left(a_{(1,1)}\right) a_{(2, \alpha)}\right) & =\sum_{(a)} A d_{r_{1}}\left(a_{(1,1)}\right) \otimes \sigma_{\alpha}\left(a_{(2, \alpha)}\right) \\
& =\sum_{(a)} A d_{r_{1}}\left(a_{(1,1)}\right) \otimes \chi_{\alpha}\left(a_{(2, \alpha)(1,1)}\right) a_{(2, \alpha)(2, \alpha)} \\
& =\sum_{(a)} A d_{r_{1}}\left(a_{(1,1)}\right) \otimes \chi_{\alpha}\left(a_{(2,1)}\right) a_{(3, \alpha)} .
\end{aligned}
$$

And because for any $a \in A_{1}, \chi_{\alpha}(a) \in k 1$, we have

$$
\begin{aligned}
\sum_{(a)} \Delta_{1, \alpha}\left(\chi_{\alpha}\left(a_{(1,1)}\right) a_{(2, \alpha)}\right) & =\sum_{(a)} \chi_{\alpha}\left(a_{(1,1)}\right) \Delta_{1, \alpha}\left(a_{(2, \alpha)}\right) \\
& =\sum_{(a)} \chi_{\alpha}\left(a_{(1,1)}\right) a_{(2, \alpha)(1,1)} \otimes a_{(2, \alpha)(2, \alpha)} \\
& =\sum_{(a)} \chi_{\alpha}\left(a_{(1,1)}\right) a_{(2,1)} \otimes a_{(3, \alpha)}, \\
\sum_{(a)} \Delta_{\alpha, 1}\left(\chi_{\alpha}\left(a_{(1,1)}\right) a_{(2, \alpha)}\right)= & \sum_{(a)} A d_{r_{\alpha}}\left(a_{(1, \alpha)}\right) \otimes \sigma_{1}\left(a_{(2,1)}\right) \\
= & \sum_{(a)} A d_{r_{\alpha}}\left(a_{(1, \alpha)}\right) \otimes \chi_{1}\left(a_{(2,1)(1,1)}\right) a_{(2,1)(2,1)} \\
= & \sum_{(a)} A d_{r_{\alpha}}\left(a_{(1, \alpha)}\right) \otimes \chi_{1}\left(a_{(2,1)}\right) a_{(3,1)},
\end{aligned}
$$


and

$$
\begin{aligned}
\sum_{(a)} \Delta_{\alpha, 1}\left(\chi_{\alpha}\left(a_{(1,1)}\right) a_{(2, \alpha)}\right) & =\sum_{(a)} \chi_{\alpha}\left(a_{(1,1)} \Delta_{\alpha, 1}\left(a_{(2, \alpha)}\right)\right. \\
& =\sum_{(a)} \chi_{\alpha}\left(a_{(1,1)} a_{(2, \alpha)(1, \alpha)} \otimes a_{(2, \alpha)(2,1)}\right. \\
& =\sum_{(a)} \chi_{\alpha}\left(a_{(1,1)}\right) a_{(2, \alpha)} \otimes a_{(3,1)}
\end{aligned}
$$

that is

$$
\sum_{(a)} A d_{r_{\alpha}}\left(a_{(1, \alpha)}\right) \otimes \chi_{1}\left(a_{(2,1)}\right) a_{(3,1)}=\sum_{(a)} \chi_{\alpha}\left(a_{(1,1)}\right) a_{(2, \alpha)} \otimes a_{(3,1)} .
$$

So we have

$$
\begin{aligned}
\sum_{(a)} A d_{r_{\alpha}}\left(a_{(1, \alpha)}\right) \chi_{1}\left(a_{(2,1)}\right) a_{(3,1)} S_{1}\left(a_{(4,1)}\right) & =\sum_{(a)} \chi_{\alpha}\left(a_{(1,1)}\right) a_{(2, \alpha)} a_{(3,1)} S_{1}\left(a_{(4,1)}\right), \\
\sum_{(a)} A d_{r_{\alpha}}\left(a_{(1, \alpha)}\right) \chi_{1}\left(a_{(2,1)}\right) & =\sum_{(a)} \chi_{\alpha}\left(a_{(1,1)}\right) a_{(2, \alpha)} .
\end{aligned}
$$

This proves (3.10). We have proved that conditions (3.9)-(3.11) are necessary conditions of the comultiplication.

On the other hand, if conditions (3.9)-(3.11) hold, then for any $a \in A_{\alpha \beta}$

$$
\begin{aligned}
\Delta_{\alpha, \beta}\left(\sigma_{\alpha \beta}(a)\right) & =\sum_{(a)} \chi\left(a_{(1,1)}\right) \Delta_{\alpha, \beta}\left(a_{(2, \alpha \beta)}\right) \\
& =\sum_{(a)} \chi\left(a_{(1,1)}\right) a_{(2, \alpha \beta)(1, \alpha)} \otimes a_{(2, \alpha \beta)(2, \beta)} \\
& =\sum_{(a)} \chi\left(a_{(1,1)}\right) a_{(2, \alpha)} \otimes a_{(3, \beta)} \\
& =\sum_{(a)} \sigma_{\alpha}\left(a_{(1, \alpha)}\right) \otimes a_{(2, \beta)}
\end{aligned}
$$

and

$$
\begin{aligned}
\Delta_{\alpha, \beta}\left(\sigma_{\alpha \beta}(a)\right) & =\sum_{(a)} \Delta_{\alpha, \beta}\left(A d_{r_{\alpha \beta}}\left(a_{(1, \alpha \beta)}\right) \chi\left(a_{(2,1)}\right)\right) \\
& =\sum_{(a)} A d_{r_{\alpha}}\left(a_{(1, \alpha \beta)(1, \alpha)}\right) \otimes A d_{r_{\beta}}\left(a_{(1, \alpha \beta)(2, \beta)}\right) \chi\left(a_{(2,1)}\right) \\
& =\sum_{(a)} A d_{r_{\alpha}}\left(a_{(1, \alpha)}\right) \otimes A d_{r_{\beta}}\left(a_{(2, \beta)}\right) \chi\left(a_{(3,1)}\right) \\
& =\sum_{(a)} A d_{r_{\alpha}}\left(a_{(1, \alpha)}\right) \otimes \sigma_{\beta}\left(a_{(2, \beta)}\right) .
\end{aligned}
$$


This proves the relations (3.14) and (3.15) hold and the comultiplication $\left.\Delta\right|_{A}$ can be extended to a homomorphism $\Delta: R \longrightarrow R \otimes R$. Since $\left(\Delta_{\alpha, \beta} \otimes\right.$ $\left.i d_{A_{\gamma}}\right) \Delta_{\alpha \beta, \gamma}(a)=\left(i d_{A_{\alpha}} \otimes \Delta_{\beta, \gamma}\right) \Delta_{\alpha, \beta \gamma}(a)$ and since $\left(\Delta_{\alpha, \beta} \otimes i d\right) \Delta_{\alpha \beta, \gamma}\left(y_{\alpha \beta \gamma}\right)=$ $\left(i d \otimes \Delta_{\beta, \gamma}\right) \Delta_{\alpha, \beta \gamma}\left(y_{\alpha \beta \gamma}\right)$ for any $\alpha, \beta, \gamma \in \pi$ and $a \in A_{\alpha \beta \gamma}$. The mapping $\Delta: R \longrightarrow R \otimes R$ is a comultiplication.

Step 2. Counit. For this part, from [6] we have known that, as $R_{1}$ admits a comultiplication, there exists a counit extending $\left.\varepsilon\right|_{A_{1}}$ and satisfying $\varepsilon\left(y_{1}\right)=0$. It follows that $\varepsilon$ admits an extension to $R$ if and only if

$$
\varepsilon\left(\delta_{1}(a)\right)=0,
$$

for any $a \in A_{1}$.

Step 3. Antipode. Let $R$ be as in Step 1. Recall that $S=\left\{S_{\alpha}: A_{\alpha} \rightarrow\right.$ $\left.A_{\alpha^{-1}}\right\}_{\alpha \in \pi}$ with $S_{\alpha}$ being an antiautomorphism and

$$
\Delta_{\beta^{-1}, \alpha^{-1}} S_{\alpha \beta}=\tau_{H_{\alpha-1}, H_{\beta^{-1}}}\left(S_{\alpha} \otimes S_{\beta}\right) \Delta_{\alpha, \beta}
$$

for any $\alpha, \beta \in \pi$. If $R$ admits an antipode $S$ which can be extended(as an antiautomorphism) from $A$ to $R$ by means of (3.8), then $S$ preserves (3.12). This means that for any $a \in A_{\alpha}$

$$
S_{\alpha}(a) S_{\alpha}\left(y_{\alpha}\right)=S_{\alpha}\left(y_{\alpha}\right) S_{\alpha} \sigma_{\alpha}(a)+S_{\alpha} \delta_{\alpha}(a) .
$$

On the other hand, if relation (3.16) holds, then $S$ can be extended as an antiautomorphism from $A$ to $R$ by means of (3.8). Using the expression $b=$ $\sum c_{i} y_{\alpha}^{i}$ of the arbitrary element $b \in R_{\alpha}$, one can readily see that the mappings $S=\left\{S_{\alpha:} R_{\alpha} \longrightarrow R_{\alpha^{-1}}\right\}$ defines above is an antipode of $R$.

Hence the existence of an antipode of $R$ satisfying (3.8) is equivalent to (3.16). It follows from (3.8) that for any $a \in A_{\alpha}$

$$
\begin{aligned}
-S_{\alpha}(a)\left(r_{\alpha^{-1}}\right)^{-1} y_{\alpha^{-1}}= & -\left(r_{\alpha^{-1}}\right)^{-1} y_{\alpha^{-1}} S_{\alpha}\left(\sigma_{\alpha}(a)\right)+S_{\alpha} \delta_{\alpha}(a), \\
-S_{\alpha}(a)\left(r_{\alpha^{-1}}\right)^{-1} y_{\alpha^{-1}}= & -\left(r_{\alpha^{-1}}\right)^{-1} \sigma_{\alpha^{-1}}\left(S_{\alpha}\left(\sigma_{\alpha}(a)\right)\right) y_{\alpha^{-1}} \\
& -\left(r_{\alpha^{-1}}\right)^{-1} \delta_{\alpha^{-1}}\left(S_{\alpha}\left(\sigma_{\alpha}(a)\right)\right)+S_{\alpha} \delta_{\alpha}(a) .
\end{aligned}
$$

Condition (3.16) holds if and only if the following two conditions hold:

$$
\begin{gathered}
S_{\alpha}(a)\left(r_{\alpha^{-1}}\right)^{-1}=\left(r_{\alpha^{-1}}\right)^{-1} \sigma_{\alpha^{-1}}\left(S_{\alpha}\left(\sigma_{\alpha}(a)\right)\right), \\
\left(r_{\alpha^{-1}}\right) S_{\alpha} \delta_{\alpha}(a)=\delta_{\alpha^{-1}}\left(S_{\alpha}\left(\sigma_{\alpha}(a)\right)\right) .
\end{gathered}
$$

Let us prove (3.17). We have

$$
\begin{aligned}
& \sigma_{\alpha^{-1}}\left(S_{\alpha}\left(\sigma_{\alpha}(a)\right)\right) \\
= & \sum_{(a)} \sigma_{\alpha^{-1}}\left(S_{\alpha}\left(\chi\left(a_{(1,1)}\right) a_{(2, \alpha)}\right)\right) \\
= & \sum_{(a)} \chi\left(a_{(1,1)}\right) \sigma_{\alpha^{-1}}\left(S_{\alpha}\left(a_{(2, \alpha)}\right)\right) \\
= & \sum_{(a)} \chi\left(a_{(1,1)}\right) A d_{\left(r_{\alpha^{-1}}\right)^{-1}}\left(\left(S_{\alpha}\left(a_{(2, \alpha)}\right)\right)_{\left(1, \alpha^{-1}\right)}\right) \chi\left(\left(S_{\alpha}\left(a_{(2, \alpha)}\right)\right)_{(2,1)}\right)
\end{aligned}
$$




$$
\begin{aligned}
& =\sum_{(a)} \chi\left(a_{(1,1)}\right) A d_{\left(r_{\alpha}-1\right)^{-1}}\left(S _ { \alpha } ( a _ { ( 2 , \alpha ) ( 2 , \alpha ) } ) \chi \left(\left(S_{1}\left(a_{(2, \alpha)(1,1)}\right)\right)\right.\right. \\
& =\sum_{(a)} \chi\left(a_{(1,1)}\right) A d_{\left(r_{\alpha-1}\right)^{-1}}\left(S _ { \alpha } ( a _ { ( 3 , \alpha ) } ) \chi \left(\left(S_{1}\left(a_{(2,1)}\right)\right)\right.\right. \\
& =\sum_{(a)} \chi\left(\varepsilon\left(a_{(1,1)}\right) 1\right) A d_{\left(r_{\alpha-1}\right)^{-1}}\left(S_{\alpha}\left(a_{(2, \alpha)}\right)\right. \\
& =A d_{\left(r_{\alpha-1}\right)^{-1}}\left(S_{\alpha}(a)\right) .
\end{aligned}
$$

Our next objective is to prove (3.18). It follows from (3.9) that we present (3.18) in an equivalent form,

$$
\left(r_{\alpha^{-1}}\right) S_{\alpha} \delta_{\alpha}(a)=\sum_{(a)} \chi\left(a_{(1,1)}\right) \delta_{\alpha^{-1}}\left(S_{\alpha}\left(a_{(2, \alpha)}\right)\right) .
$$

We denote $L_{\alpha}=\left(r_{\alpha^{-1}}\right) S_{\alpha} \delta_{\alpha}(a)$ and $M_{\alpha}=\sum_{(a)} \chi\left(a_{(1,1)}\right) \delta_{\alpha^{-1}}\left(S_{\alpha}\left(a_{(2, \alpha)}\right)\right)$.

From (3.11) we have

$$
\Delta_{\alpha, \alpha^{-1}}\left(\delta_{1}(a)\right)=\sum_{(a)} \delta_{\alpha}\left(a_{(1, \alpha)}\right) \otimes a_{\left(2, \alpha^{-1}\right)}+r_{\alpha} a_{(1, \alpha)} \otimes \delta_{\alpha^{-1}}\left(a_{\left(2, \alpha^{-1}\right)}\right),
$$

and we apply $m\left(i d \otimes S_{\alpha^{-1}}\right)$ to the above equality, we get

$$
\begin{aligned}
& m\left(i d \otimes S_{\alpha^{-1}}\right)\left(\Delta_{\alpha, \alpha^{-1}}\left(\delta_{1}(a)\right)\right) \\
= & m\left(i d \otimes S_{\alpha^{-1}}\right)\left(\sum_{(a)} \delta_{\alpha}\left(a_{(1, \alpha)}\right) \otimes a_{\left(2, \alpha^{-1}\right)}+r_{\alpha} a_{(1, \alpha)} \otimes \delta_{\alpha^{-1}}\left(a_{\left(2, \alpha^{-1}\right)}\right)\right), \\
0= & \left.\varepsilon\left(\delta_{1}(a)\right)\right) 1_{\alpha} \\
= & \sum_{(a)} \delta_{\alpha}\left(a_{(1, \alpha)}\right) S_{\alpha^{-1}}\left(a_{\left(2, \alpha^{-1}\right)}\right)+r_{\alpha} a_{(1, \alpha)} S_{\alpha^{-1}}\left(\delta_{\alpha^{-1}}\left(a_{\left(2, \alpha^{-1}\right)}\right)\right), \\
& -\left(r_{\alpha}\right)^{-1} \sum_{(a)} \delta_{\alpha}\left(a_{(1, \alpha)}\right) S_{\alpha^{-1}}\left(a_{\left(2, \alpha^{-1}\right)}\right)=\sum_{(a)} a_{(1, \alpha)} S_{\alpha^{-1}}\left(\delta_{\alpha^{-1}}\left(a_{\left(2, \alpha^{-1}\right)}\right)\right) .
\end{aligned}
$$

Then for any $a \in A_{\alpha^{-1}}$

$$
\begin{aligned}
L_{\alpha^{-1}} & =r_{\alpha} S_{\alpha^{-1}} \delta_{\alpha^{-1}}(a)=\sum_{(a)} r_{\alpha} \varepsilon\left(a_{(1,1)}\right) S_{\alpha^{-1}} \delta_{\alpha^{-1}}\left(a_{\left(2, \alpha^{-1}\right)}\right) \\
& =\sum_{(a)} r_{\alpha} S_{\alpha^{-1}}\left(a_{\left(1, \alpha^{-1}\right)}\right) a_{(2, \alpha)} S_{\alpha^{-1}} \delta_{\alpha^{-1}}\left(a_{\left(3, \alpha^{-1}\right)}\right) \\
& =\sum_{(a)}-r_{\alpha} S_{\alpha^{-1}}\left(a_{\left(1, \alpha^{-1}\right)}\right)\left(r_{\alpha}\right)^{-1} \delta_{\alpha}\left(a_{(2, \alpha)}\right) S_{\alpha^{-1}}\left(a_{\left(3, \alpha^{-1}\right)}\right) \\
& =\sum_{(a)}-A d_{r_{\alpha}}\left(S_{\alpha^{-1}}\left(a_{\left(1, \alpha^{-1}\right)}\right)\right) \delta_{\alpha}\left(a_{(2, \alpha)}\right) S_{\alpha^{-1}}\left(a_{\left(3, \alpha^{-1}\right)}\right) .
\end{aligned}
$$


On the other hand, for any $a \in A_{1}$, we have $\varepsilon(a) 1_{\alpha}=\sum_{(a)} a_{(1, \alpha)} S_{\alpha^{-1}}\left(a_{\left(2, \alpha^{-1}\right)}\right)$. The action by $\delta_{\alpha}$ on both sides gives

$$
0=\sum_{(a)} \delta_{\alpha}\left(a_{(1, \alpha)}\right) S_{\alpha^{-1}}\left(a_{\left(2, \alpha^{-1}\right)}\right)+\sigma_{\alpha}\left(a_{(1, \alpha)}\right) \delta_{\alpha} S_{\alpha^{-1}}\left(a_{\left(2, \alpha^{-1}\right)}\right),
$$

$$
\begin{aligned}
& M_{\alpha^{-1}} \\
= & \sum_{(a)} \chi\left(a_{(1,1)}\right) \delta_{\alpha}\left(S_{\alpha^{-1}}\left(a_{\left(2, \alpha^{-1}\right)}\right)\right) \\
= & \sum_{(a)} \chi\left(a_{(1,1)}\right) \varepsilon\left(a_{(2,1)}\right) \delta_{\alpha}\left(S_{\alpha^{-1}}\left(a_{\left(3, \alpha^{-1}\right)}\right)\right) \\
= & \sum_{(a)} \chi\left(a_{(1,1)}\right) \sigma_{\alpha}\left(S_{\alpha^{-1}}\left(a_{\left(2, \alpha^{-1}\right)}\right) a_{(3, \alpha)}\right) \delta_{\alpha}\left(S_{\alpha^{-1}}\left(a_{\left(4, \alpha^{-1}\right)}\right)\right) \\
= & \sum_{(a)} \chi\left(a_{(1,1)}\right) \sigma_{\alpha}\left(S_{\alpha^{-1}}\left(a_{\left(2, \alpha^{-1}\right)}\right)\right) \sigma_{\alpha}\left(a_{(3, \alpha)}\right) \delta_{\alpha}\left(S_{\alpha^{-1}}\left(a_{\left(4, \alpha^{-1}\right.}\right)\right) \\
= & -\sum_{(a)} \chi\left(a_{(1,1)}\right) \sigma_{\alpha}\left(S_{\alpha^{-1}}\left(a_{\left(2, \alpha^{-1}\right)}\right)\right) \delta_{\alpha}\left(a_{(3, \alpha)}\right) S_{\alpha^{-1}}\left(a_{\left(4, \alpha^{-1}\right)}\right) \\
= & -\sum_{(a)} \chi\left(a_{(1,1)}\right) A d_{r_{\alpha}}\left(S_{\alpha^{-1}}\left(a_{\left(2, \alpha^{-1}\right)}\right)(1, \alpha)\right) \chi\left(S_{\alpha^{-1}}\left(a_{\left(2, \alpha^{-1}\right)}\right)(2,1)\right) \delta_{\alpha}\left(a_{(3, \alpha)}\right) S_{\alpha^{-1}}\left(a_{\left(4, \alpha^{-1}\right)}\right) \\
= & -\sum_{(a)} \chi\left(a_{(1,1)}\right) A d_{r_{\alpha}}\left(S_{\alpha^{-1}}\left(a_{\left(3, \alpha^{-1}\right)}\right)\right) \chi\left(S_{1}\left(a_{(2,1)}\right)\right) \delta_{\alpha}\left(a_{(4, \alpha)}\right) S_{\alpha^{-1}}\left(a_{\left(5, \alpha^{-1}\right)}\right) \\
= & -\sum_{(a)} \chi\left(\varepsilon\left(a_{(1,1)}\right) 1\right) A d_{r_{\alpha}}\left(S_{\alpha^{-1}}\left(a_{\left(2, \alpha^{-1}\right)}\right)\right) \delta_{\alpha}\left(a_{(3, \alpha)}\right) S_{\alpha^{-1}}\left(a_{\left(4, \alpha^{-1}\right)}\right) \\
= & -\sum_{(a)} A d_{r_{\alpha}}\left(S_{\alpha^{-1}}\left(a_{\left(1, \alpha^{-1}\right)}\right)\right) \delta_{\alpha}\left(a_{(2, \alpha)}\right) S_{\alpha^{-1}}\left(a_{\left(3, \alpha^{-1}\right)}\right) .
\end{aligned}
$$

Comparing (3.20) and (3.21) we conclude that $L_{\alpha}=M_{\alpha}$. This proves both relation (3.19) and the existence of an antipode.

Here we give an example from [12], and ours is a little different, where the condition crossing is not necessarily needed.

Example 3.6. For $n \in \mathbb{Z}$ and $\alpha=\left(\alpha_{i j}\right), \beta=\left(\beta_{i j}\right) \in G L_{n}(k)$, let $B_{n}^{(\alpha, \beta)}$ be the algebra generalized by symbols $g, x_{1}, \ldots, x_{n}$ satisfying the following relations: for $i \in\{1,2, \ldots, n\}$,

$$
g^{2}=1, \quad x_{i}^{2}=0, \quad g x_{i}=-x_{i} g, \quad x_{i} x_{j}=-x_{j} x_{i} .
$$

The family of algebras $\mathbb{D}_{n}=\left\{B_{n}^{(\alpha, \beta)}\right\}_{(\alpha, \beta) \in \varsigma\left(G L_{n}(k)\right)}$ has a structure of $\varsigma\left(G L_{n}(k)\right)$ coalgebra given, for any $\alpha=\left(\alpha_{i j}\right), \beta=\left(\beta_{i j}\right), \lambda=\left(\lambda_{i j}\right), \gamma=\left(\gamma_{i j}\right) \in \varsigma\left(G L_{n}(k)\right)$, and $i \leq i \leq n$, by

$$
\begin{gathered}
\Delta_{(\alpha, \beta),(\lambda, \gamma)}(g)=g \otimes g, \\
\Delta_{(\alpha, \beta),(\lambda, \gamma)}\left(x_{i}\right)=\sum_{k=1}^{n} \tilde{\gamma}_{k, i} x_{k} \otimes 1+1 \otimes \sum_{k=1, i=1, p=1}^{n} \gamma_{k, i} \tilde{\alpha}_{i, p} \tilde{\gamma}_{p, k} x_{k},
\end{gathered}
$$




$$
\begin{gathered}
\varepsilon(g)=1, \quad \varepsilon\left(x_{i}\right)=0 \\
S_{(\alpha, \beta)}(g)=g, \quad S_{(\alpha, \beta)}\left(x_{i}\right)=\sum_{k=1, p=1}^{n} \tilde{\beta}_{k, j} \tilde{\alpha}_{j, i} g x_{k},
\end{gathered}
$$

where $\left(\tilde{\alpha}_{i, j}\right)=\alpha^{-1}$ for any $\alpha \in G L_{n}(k)$.

Now we will add $n$ indeterminates $y_{1}, \ldots, y_{n}$ by Ore extensions. Firstly, define $\sigma_{1}, \delta_{1}: B_{n}^{(\alpha, \beta)} \rightarrow B_{n}^{(\alpha, \beta)}$ by

$$
\sigma_{1}(g)=-g, \sigma_{1}\left(x_{i}\right)=x_{i}
$$

and

$$
\delta_{1}(g)=0, \delta_{1}\left(x_{i}\right)=\left(\alpha_{1 j}-\beta_{1 j}\right) g .
$$

It is easy to check that $\sigma_{1}$ is an endomorphism of $B_{n}^{(\alpha, \beta)}$ and $\delta_{1}$ is a $\sigma_{1}$ derivation. Thus we get the Ore extension $B_{n, 1}^{(\alpha, \beta)}=B_{n}^{(\alpha, \beta)}\left[y_{1} ; \sigma_{1}, \delta_{1}\right]$. Define

$$
\Delta_{(\alpha, \beta),(\lambda, \gamma)}\left(y_{1}\right)=y_{1} \otimes 1+g \otimes y_{1},
$$

then $B_{n, 1}^{(\alpha, \beta)}\left[y_{1} ; \sigma_{1}, \delta_{1}\right]$ is the Hopf group coalgebra Ore extension of $B_{n}$.

Then we define $\sigma_{2}, \delta_{2}: B_{n, 1}^{(\alpha, \beta)} \rightarrow B_{n, 1}^{(\alpha, \beta)}$ by

$$
\sigma_{2}(g)=-g, \sigma_{2}\left(x_{i}\right)=x_{i}, \sigma_{2}\left(y_{1}\right)=-y_{1}
$$

and

$$
\delta_{2}(g)=0, \delta_{2}\left(x_{i}\right)=\left(\alpha_{2 j}-\beta_{2 j}\right) g, \delta_{2}\left(y_{1}\right)=0 .
$$

It is easy to check that $\sigma_{1}$ is an endomorphism of $B_{n, 1}^{(\alpha, \beta)}$ and $\delta_{2}$ is a $\sigma_{2}$ derivation. Thus we get the Ore extension $B_{n, 2}^{(\alpha, \beta)}=B_{n, 1}^{(\alpha, \beta)}\left[y_{1} ; \sigma_{1}, \delta_{1}\right]$. When we define

$$
\Delta_{(\alpha, \beta),(\lambda, \gamma)}\left(y_{2}\right)=y_{2} \otimes 1+g \otimes y_{2},
$$

$B_{n, 2}^{(\alpha, \beta)}$ is also an Hopf group coalgebra Ore extension of $B_{n, 1}$.

We continue the process by $n$ times, then we will add $n$ indeterminates and get the Hopf group coalgebra $A_{n}^{(\alpha, \beta)}$ as in the example in [12].

\section{Isomophism}

In this section, we study the relations of two Hopf group coalgebra Ore extensions. First we need to give the following lemma.

Lemma 4.1 ([1, Lemma 1.1]). Let $A$ be a algebra, $A[y ; \sigma, \delta]$ an Ore extension of $A$ and $i: A \rightarrow A[y ; \sigma, \delta]$ the inclusion morphism. Then for any algebra $B$, any algebra morphism $f: A \rightarrow B$ and every element $b \in B$ such that $b f(a)=$ 
$f(\delta(a))+f(\sigma(a)) b$ for any $a \in A$, there exists a unique algebra morphism $\bar{f}: A[y ; \sigma, \delta] \rightarrow B$ such that $\bar{f}(y)=b$ and the following diagram is commutative:

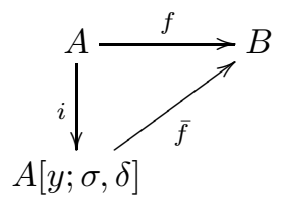

Similarly, we can generalized this lemma to the cases of $\pi$-graded algebras:

Lemma 4.2. Let $A=\left\{A_{\alpha}\right\}_{\alpha \in \pi}$ be a $\pi$-graded algebra, $R=\left\{R_{\alpha}=A_{\alpha}\left[y_{\alpha} ; \sigma \alpha, \delta_{\alpha}\right]\right\}$ the Ore extension of $A$, and $i: A \rightarrow R$ the inclusion morphism, where $i=\left\{i_{\alpha}\right.$ : $\left.A_{\alpha} \rightarrow A_{\alpha}\left[y_{\alpha} ; \sigma \alpha, \delta_{\alpha}\right]\right\}$. For any $\pi$-graded algebra $B=\left\{B_{\alpha}\right\}_{\alpha \in \pi}, f: A \rightarrow B$ algebra morphism, where $f=\left\{f_{\alpha}: A_{\alpha} \rightarrow B_{\alpha}\right\}_{\alpha \in \pi}$ and $f_{\alpha}$ is an algebra morphism. Then for any element $b=\left\{b_{\alpha}\right\}_{\alpha \in \pi} \in B$, if for any $\alpha \in \pi$, $b_{\alpha} f_{\alpha}\left(a_{\alpha}\right)=f_{\alpha}\left(\delta_{\alpha}\left(a_{\alpha}\right)\right)+f\left(\sigma_{\alpha}\left(a_{\alpha}\right)\right) b_{\alpha}$ for any $a=\left\{a_{\alpha}\right\} \in A$, then there exists a unique algebra morphism $\bar{f}=\left\{\bar{f}_{\alpha}\right\}: R \rightarrow B$ such that $\bar{f}_{\alpha}\left(y_{\alpha}\right)=b_{\alpha}$ and the following diagram is commutative:

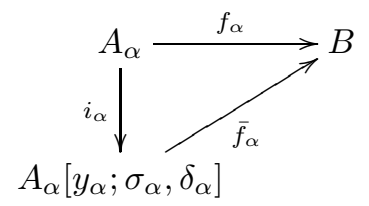

Proof. The proof is simple. By Lemma 4.1 we can get the result directly.

Definition 4.3. Let $A=\left\{A_{\alpha}\right\}$ and $A^{\prime}=\left\{A_{\alpha}^{\prime}\right\}$ be two Hopf group coalgebras, we call $f: A \rightarrow A^{\prime}$, where $f=\left\{f_{\alpha}\right\}$ and $f_{\alpha}: A_{\alpha} \rightarrow A_{\alpha}^{\prime}$, the morphism of Hopf group coalgebra if for any $\alpha \in \pi, f_{\alpha}$ is algebra morphism, $\Delta_{\alpha, \beta}^{\prime} f_{\alpha \beta}=$ $\left(f_{\alpha} \otimes f_{\beta}\right) \Delta_{\alpha, \beta}$ and $S_{\alpha}^{\prime} f_{\alpha}=f_{\alpha^{-1}} S_{\alpha}$, where $\Delta, \Delta^{\prime}, S, S^{\prime}$ are the comultiplications and antipodes of $A$ and $A^{\prime}$ respectively.

Obviously we can see that this definition generalizes the notion of morphisms of Hopf algebra, and when $\pi=1$, it is the usual Hopf algebra morphism.

In order to simplify the notation and study the isomorphism of Hopf group coalgebra-Ore extensions, we introduce the following definitions.

Definition 4.4. Let $A=\left\{A_{\alpha}\right\}$ be a Hopf group coalgebra. Denote $r=$ $\left\{r_{\alpha}\right\}, \sigma=\left\{\sigma_{\alpha}\right\}$. A family of mappings $\delta=\left\{\delta_{\alpha}\right\}$ satisfying (3.11) is called a $r$-coderivation. If $\delta$ is also a $\sigma$-derivation where $\sigma$ is an algebra morphism satisfying (3.9) and (3.10), then $\delta$ is called a $\langle\chi, r\rangle$-derivation.

Notation. Denote the Hopf group coalgebra-Ore extension $R=\left\{R_{\alpha}\right\}_{\alpha \in \pi}$ by $R=\left\{R_{\alpha}=A_{\alpha}\left(\chi, r_{\alpha}, \delta_{\alpha}\right)\right\}_{\alpha \in \pi}$, where $\chi: A_{1} \rightarrow k$ is a character, $r=\left\{r_{\alpha}\right\}_{\alpha \in \pi}$ is a family of group-like element of $A$ and $\delta$ is a $\langle\chi, r\rangle$-derivation.

Now we define an isomorphism of Hopf group coalgebra-Ore extensions. 
Definition 4.5. Two Hopf group coalgebra-Ore extensions

$$
R=\left\{R_{\alpha}=A_{\alpha}\left(\chi, r_{\alpha}, \delta_{\alpha}\right)\right\}_{\alpha \in \pi} \text { and } R^{\prime}=\left\{R_{\alpha}^{\prime}=A_{\alpha}^{\prime}\left(\chi, r_{\alpha}^{\prime}, \delta_{\alpha}^{\prime}\right)\right\}_{\alpha \in \pi}
$$

of Hopf group coalgebras $A$ and $A^{\prime}$ are said to be isomorphism if there is an isomorphism of Hopf group coalgebras $\phi: R \rightarrow R^{\prime}$ such that $\phi(A)=A^{\prime}$.

Remark. Actually the isomorphism of Hopf group coalgebras $\phi$ is a family of isomorphism of algebras $\left\{\phi_{\alpha}: A_{\alpha} \rightarrow A_{\alpha}^{\prime}\right\}_{\alpha \in \pi}$ and satisfies the conditions in Definition 4.3.

Definition 4.6. A $\langle\chi, r\rangle$-derivation $\delta=\left\{\delta_{\alpha}\right\}$ is inner, where $r=\left\{r_{\alpha}\right\}_{\alpha \in \pi}$ if there is a family of elements $\left\{d_{\alpha}\right\}_{\alpha \in \pi} \in A$ such that for all $a \in A_{\alpha}, \delta_{\alpha}(a)=$ $\sigma_{\alpha}(a) d_{\alpha}-d_{\alpha} a$ and $\Delta_{\alpha, \beta}\left(d_{\alpha \beta}\right)=d_{\alpha} \otimes 1+r_{\alpha} \otimes d_{\beta}$.

Lemma 4.7. If $\delta$ is a $\langle\chi, r\rangle$-derivation, then we have

$$
\varepsilon\left(d_{1}\right)=0
$$

and

$$
S_{\alpha}\left(d_{\alpha}\right)=-\left(r_{\alpha^{-1}}\right)^{-1} d_{\alpha^{-1}}
$$

Proof. By Definition 4.6, $\Delta_{1,1}\left(d_{1}\right)=d_{1} \otimes 1+r_{1} \otimes d_{1}$, then we have $d_{1}=\varepsilon\left(d_{1}\right) 1+$ $\varepsilon\left(r_{1}\right) d_{1}$. So by $\varepsilon\left(r_{1}\right)=1$ we get $\varepsilon\left(d_{1}\right)=0$. And $\Delta_{\alpha, \alpha^{-1}}\left(d_{1}\right)=d_{\alpha} \otimes 1+r_{\alpha} \otimes d_{\alpha^{-1}}$, so $0=\varepsilon\left(d_{1}\right) 1_{\alpha^{-1}}=S_{\alpha}\left(d_{\alpha}\right)+S_{\alpha}\left(r_{\alpha}\right) d_{\alpha^{-1}}=S_{\alpha}\left(d_{\alpha}\right)+\left(r_{\alpha^{-1}}\right)^{-1} d_{\alpha^{-1}}$, then we get $S_{\alpha}\left(d_{\alpha}\right)=-\left(r_{\alpha^{-1}}\right)^{-1} d_{\alpha^{-1}}$ for any $\alpha \in \pi$.

Using the above lemma, we prove the following consequence for an inner $\langle\chi, r\rangle$-derivation.

Proposition 4.8. Let $R=\left\{R_{\alpha}=A_{\alpha}\left(\chi, r_{\alpha}, \delta_{\alpha}\right)\right\}_{\alpha \in \pi}$ be a Hopf group coalgebraOre extension of $A$. If $\delta=\left\{\delta_{\alpha}\right\}$ is an inner $\langle\chi, r\rangle$-derivation, then $R=\left\{R_{\alpha}=\right.$ $\left.A_{\alpha}\left(\chi, r_{\alpha}, \delta_{\alpha}\right)\right\}_{\alpha \in \pi}$ is isomorphism to the Hopf group coalgebra-Ore extension $R=\left\{R_{\alpha}=A_{\alpha}\left(\chi, r_{\alpha}, 0\right)\right\}_{\alpha \in \pi}$.

Proof. Denote the indeterminates of $\left\{A_{\alpha}\left(\chi, r_{\alpha}, \delta_{\alpha}\right)\right\}_{\alpha \in \pi}$ and $\left\{A_{\alpha}\left(\chi, r_{\alpha}, 0\right)\right\}_{\alpha \in \pi}$ by $y=\left\{y_{\alpha}\right\}_{\alpha \in \pi}$ and $y^{\prime}=\left\{y_{\alpha}^{\prime}\right\}_{\alpha \in \pi}$. Firstly we uniquely extend the inclusion morphism $i=\left\{i_{\alpha}\right\}: A \rightarrow\left\{A_{\alpha}\left(\chi, r_{\alpha}, 0\right)\right\}_{\alpha \in \pi}$ to the algebra morphism $\left\{A_{\alpha}\left(\chi, r_{\alpha}, \delta_{\alpha}\right)\right\}_{\alpha \in \pi} \rightarrow\left\{A_{\alpha}\left(\chi, r_{\alpha}, 0\right)\right\}_{\alpha \in \pi}$ by extending $\left.i_{\alpha}: A_{\alpha} \rightarrow A_{\alpha}\left(\chi, r_{\alpha}, 0\right)\right\}$ for any $\alpha \in \pi$ which is denoted by $\bar{i}$ and $\bar{i}_{\alpha}$ such that the following diagram is commutative:

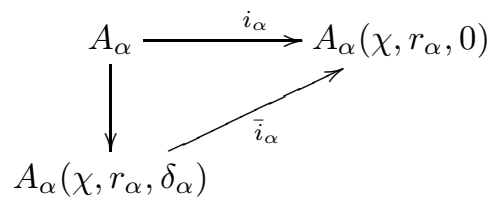


To see this, take $\left\{y_{\alpha}^{\prime}-d_{\alpha}\right\} \in\left\{A_{\alpha}\left(\chi, r_{\alpha}, 0\right)\right\}_{\alpha \in \pi}$ and define $\bar{i}_{\alpha}\left(y_{\alpha}\right)=y_{\alpha}^{\prime}-d_{\alpha}$. Because $\delta=\left\{\delta_{\alpha}\right\}$ is inner, we have for all $a \in A_{\alpha}$

$$
\begin{aligned}
i_{\alpha} \sigma_{\alpha}(a) \bar{i}_{\alpha}\left(y_{\alpha}\right)+i_{\alpha} \delta_{\alpha}(a) & =\sigma_{\alpha}(a)\left(y_{\alpha}^{\prime}-d_{\alpha}\right)+\delta_{\alpha}(a) \\
& =\sigma_{\alpha}(a) y_{\alpha}^{\prime}-\sigma_{\alpha}(a) d_{\alpha}+\delta_{\alpha}(a) \\
& =\sigma_{\alpha}(a) y_{\alpha}^{\prime}-\sigma_{\alpha}(a) d_{\alpha}+\sigma_{\alpha}(a) d_{\alpha}-d_{\alpha} a \\
& =\sigma_{\alpha}(a) y_{\alpha}^{\prime}-d_{\alpha} a \\
& =\left(y_{\alpha}^{\prime}-d_{\alpha}\right) a \\
& =\left(y_{\alpha}^{\prime}-d_{\alpha}\right) i_{\alpha}(a) .
\end{aligned}
$$

Then by Lemma 4.2, we complete the extension.

Similarly, the inclusion morphism $j: A \rightarrow\left\{A_{\alpha}\left(\chi, r_{\alpha}, \delta_{\alpha}\right)\right\}_{\alpha \in \pi}$ can be uniquely extended to the algebra morphism $\bar{j}:\left\{A_{\alpha}\left(\chi, r_{\alpha}, 0\right)\right\}_{\alpha \in \pi} \rightarrow\left\{A_{\alpha}\left(\chi, r_{\alpha}, \delta_{\alpha}\right)\right\}_{\alpha \in \pi}$. By the uniqueness of extension, we get that $\bar{i}$ is an algebra morphism and $\bar{j}$ is its inverse.

It is easy to verify that $\bar{i}_{\alpha}\left(A_{\alpha}\left(\chi, r_{\alpha}, \delta_{\alpha}\right)\right) \subseteq A_{\alpha}\left(\chi, r_{\alpha}, 0\right)$, and so we have constructed an algebra isomorphism from $\left\{A_{\alpha}\left(\chi, r_{\alpha}, \delta_{\alpha}\right)\right\}_{\alpha \in \pi}$ to $\left\{A_{\alpha}\left(\chi, r_{\alpha}, 0\right)\right\}_{\alpha \in \pi}$ satisfying $\bar{i}(A)=A$.

By the definitions of an inner $\langle\chi, r\rangle$-derivation and a Hopf group coalgebraOre extension, we have the calculation

$$
\begin{aligned}
\left(\bar{i}_{\alpha} \otimes \bar{i}_{\beta}\right) \Delta_{\alpha, \beta}\left(y_{\alpha \beta}\right) & =\left(\bar{i}_{\alpha} \otimes \bar{i}_{\beta}\right)\left(y_{\alpha} \otimes 1+r_{\alpha} \otimes y_{\beta}\right) \\
& =\bar{i}\left(y_{\alpha}\right) \otimes 1+r_{\alpha} \otimes \bar{i}_{\beta}\left(y_{\beta}\right) \\
& =\left(y_{\alpha}^{\prime}-d_{\alpha}\right) \otimes 1+r_{\alpha} \otimes\left(y_{\beta}^{\prime}-d_{\beta}\right) \\
& =y_{\alpha}^{\prime} \otimes 1+r_{\alpha} \otimes y_{\beta}^{\prime}-d_{\alpha} \otimes 1-r_{\alpha} \otimes d_{\beta} \\
& =y_{\alpha}^{\prime} \otimes 1+r_{\alpha} \otimes y_{\beta}^{\prime}-\Delta_{\alpha, \beta} d_{\alpha \beta} \\
& =\Delta_{\alpha, \beta}^{\prime}\left(y_{\alpha \beta}^{\prime}\right)-\Delta_{\alpha, \beta}^{\prime}\left(d_{\alpha \beta}\right) \\
& =\Delta_{\alpha, \beta}^{\prime}\left(y_{\alpha \beta}^{\prime}-d_{\alpha \beta}\right) \\
& =\Delta_{\alpha, \beta}^{\prime} \bar{i}_{\alpha \beta}\left(y_{\alpha \beta}\right) .
\end{aligned}
$$

And

$$
\begin{aligned}
\bar{i}_{\alpha} S_{\alpha}\left(y_{\alpha}\right) & =\bar{i}_{\alpha}\left(-\left(r_{\alpha^{-1}}\right)^{-1} y_{\alpha^{-1}}\right) \\
& =-\left(r_{\alpha^{-1}}\right)^{-1}\left(y_{\alpha^{-1}}^{\prime}-d_{\alpha^{-1}}\right) \\
& =-\left(r_{\alpha^{-1}}\right)^{-1} y_{\alpha^{-1}}^{\prime}+\left(r_{\alpha^{-1}}\right)^{-1} d_{\alpha^{-1}} \\
& =-\left(r_{\alpha^{-1}}\right)^{-1} y_{\alpha^{-1}}^{\prime}-S_{\alpha}\left(d_{\alpha}\right) \\
& =S_{\alpha}^{\prime}\left(y_{\alpha}^{\prime}\right)-S_{\alpha}^{\prime}\left(d_{\alpha}\right) \\
& =S_{\alpha}^{\prime}\left(y_{\alpha}^{\prime}-d_{\alpha}\right) \\
& =S_{\alpha}^{\prime} \bar{i}_{\alpha}\left(y_{\alpha}\right) .
\end{aligned}
$$

Thus we proves the proposition. 
Now we can introduce and prove the main result in this section.

Theorem 4.9. Let $R=\left\{A_{\alpha}\left(\chi, r_{\alpha}, \delta_{\alpha}\right)\right\}_{\alpha \in \pi}$ and $R^{\prime}=\left\{A_{\alpha}^{\prime}\left(\chi^{\prime}, r_{\alpha}^{\prime}, \delta_{\alpha}^{\prime}\right)\right\}_{\alpha \in \pi}$ be two Hopf group coalgebra-Ore extensions. If there exists a isomorphism of Hopf group coalgebra $\phi: R \rightarrow R^{\prime}$ such that $\chi^{\prime}=\chi \phi^{-1}, r^{\prime}=\phi(r)$ and $\delta^{\prime}=\phi \delta \phi^{-1}+\delta^{\prime \prime}$, where $r=\left\{r_{\alpha}\right\}, r^{\prime}=\left\{r_{\alpha}^{\prime}\right\}, \delta=\left\{\delta_{\alpha}\right\}, \delta^{\prime}=\left\{\delta_{\alpha}^{\prime}\right\}$ and $\delta^{\prime \prime}$ is an inner $\left\langle\chi^{\prime}, r^{\prime}\right\rangle$-derivation of $A^{\prime}$, then $R$ is isomorphism to $R^{\prime}$ as a Hopf group coalgebra-Ore extension.

Proof. Denote the indeterminates of $\left\{A_{\alpha}\left(\chi, r_{\alpha}, \delta_{\alpha}\right)\right\}_{\alpha \in \pi}$ and $\left\{A_{\alpha}^{\prime}\left(\chi^{\prime}, r_{\alpha}^{\prime}, \delta_{\alpha}^{\prime}\right)\right\}_{\alpha \in \pi}$ by $y=\left\{y_{\alpha}\right\}_{\alpha \in \pi}$ and $y^{\prime}=\left\{y_{\alpha}^{\prime}\right\}_{\alpha \in \pi}$. We will show that there exist extensions of $\phi$ and $\phi^{-1}$, which are denoted by $\bar{\phi}$ and $\phi^{-1}$, such that the following diagrams are commutative:

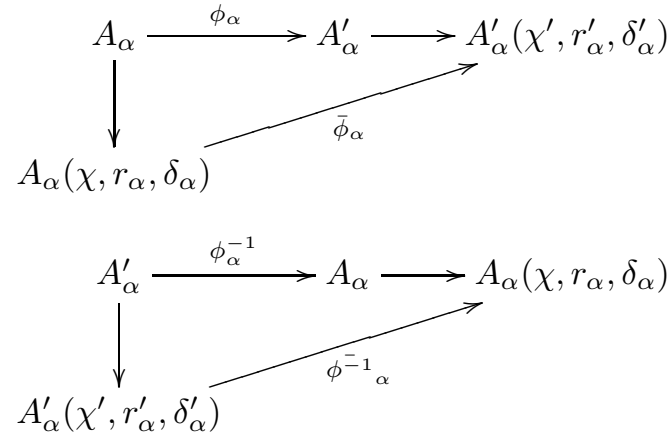

To prove this, we first have to show $\sigma_{\alpha}^{\prime} \phi_{\alpha}=\phi_{\alpha} \sigma_{\alpha}, \delta_{\alpha}^{\prime} \phi_{\alpha}=\phi_{\alpha} \delta_{\alpha}, \sigma_{\alpha} \phi^{-1}=$ $\phi^{-1} \sigma_{\alpha}^{\prime}$ and $\delta_{\alpha} \phi^{-1}=\phi^{-1} \delta_{\alpha}^{\prime}$. In fact, by the assumption, for all $a \in A_{\alpha}$, we have

$$
\begin{aligned}
\sigma_{\alpha}^{\prime} \phi_{\alpha}(a) & =\sum_{(a)} \chi^{\prime}\left(\phi_{\alpha}(a)_{(1,1)}\right) \phi_{\alpha}(a)_{(2, \alpha)}=\sum_{(a)} \chi^{\prime}\left(\phi_{\alpha}\left(a_{(1,1)}\right)\right) \phi_{\alpha}\left(a_{(2, \alpha))}\right. \\
& =\sum_{(a)} \chi\left(\phi_{\alpha}^{-1} \phi_{\alpha}\left(a_{(1,1)}\right)\right) \phi_{\alpha}\left(a_{(2, \alpha))}=\sum_{(a)} \chi\left(a_{(1,1)}\right) \phi_{\alpha}\left(a_{(2, \alpha))}\right.\right. \\
& =\sum_{(a)} \phi_{\alpha}\left(\chi\left(a_{(1,1)}\right) \phi_{\alpha}\left(a_{(2, \alpha))}\right)=\phi_{\alpha} \sigma_{\alpha}(a)\right.
\end{aligned}
$$

Similarly for $\delta_{\alpha}^{\prime} \phi_{\alpha}=\phi_{\alpha} \delta_{\alpha}$. We can prove the other two equations directly by $\delta_{\alpha}^{\prime}=\phi_{\alpha} \delta_{\alpha} \phi_{\alpha}^{-1}$.

Then we have

$$
y_{\alpha}^{\prime} \phi_{\alpha}(a)=\sigma_{\alpha}^{\prime}\left(\phi_{\alpha}(a)\right) y_{\alpha}^{\prime}+\delta_{\alpha}^{\prime}\left(\phi_{\alpha}(a)\right)=\phi_{\alpha} \sigma_{\alpha}(a) y_{\alpha}^{\prime}+\phi_{\alpha} \delta_{\alpha}(a)
$$

for all $a \in A_{\alpha}$ and

$$
y_{\alpha} \phi_{\alpha}^{-1}\left(a^{\prime}\right)=\sigma_{\alpha}\left(\phi_{\alpha}^{-1}\left(a^{\prime}\right)\right) y_{\alpha}+\delta_{\alpha}\left(\phi_{\alpha}^{-1}\left(a^{\prime}\right)\right)=\left(\phi_{\alpha}^{-1} \sigma_{\alpha}^{\prime}\left(a^{\prime}\right)\right) y_{\alpha}+\phi_{\alpha}^{-1} \delta_{\alpha}^{\prime}\left(a^{\prime}\right)
$$

for all $a^{\prime} \in A_{\alpha}^{\prime}$. So $\bar{\phi}$ and $\phi^{-1}$ are extensions of $\phi$ and $\phi^{-1}$, respectively, such that $\bar{\phi}_{\alpha}\left(y_{\alpha}\right)=y_{\alpha}^{\prime}$ and $\phi^{-1}{ }_{\alpha}\left(y_{\alpha}^{\prime}\right)=y_{\alpha}$. And by the uniqueness of the extensions, we obtain that $\phi^{-1} \circ \bar{\phi}=i d$ and $\bar{\phi} \circ \phi^{-1}=i d$. 
Using the assumption $r^{\prime}=\phi(r)$, we have

$$
\begin{aligned}
\left(\bar{\phi}_{\alpha} \otimes \bar{\phi}_{\beta}\right) \Delta_{\alpha, \beta}\left(y_{\alpha \beta}\right) & =\left(\bar{\phi}_{\alpha} \otimes \bar{\phi}_{\beta}\right)\left(y_{\alpha} \otimes 1+r_{\alpha} \otimes y_{\beta}\right) \\
& =y_{\alpha}^{\prime} \otimes 1+\phi_{\alpha}\left(r_{\alpha}\right) \otimes y_{\beta}^{\prime} \\
& =y_{\alpha}^{\prime} \otimes 1+r_{\alpha}^{\prime} \otimes y_{\beta}^{\prime} \\
& =\Delta_{\alpha, \beta}^{\prime}\left(y_{\alpha \beta}^{\prime}\right) \\
& =\Delta_{\alpha, \beta}^{\prime} \bar{\phi}_{\beta}\left(y_{\alpha \beta}\right) .
\end{aligned}
$$

Similarly for $\Delta_{\alpha, \beta} \phi_{\alpha \beta}^{-1}\left(y_{\alpha \beta}^{\prime}\right)=\left(\phi_{\alpha}^{-1} \otimes \phi_{\beta}^{-1}\right) \Delta_{\alpha, \beta}^{\prime}\left(y_{\alpha \beta}^{\prime}\right)$. And

$$
\begin{aligned}
S_{\alpha}^{\prime} \bar{\phi}_{\alpha}\left(y_{\alpha}\right) & =S_{\alpha}^{\prime}\left(y_{\alpha}^{\prime}\right)=-\left(r_{\alpha^{-1}}^{\prime}\right)^{-1} y_{\alpha^{-1}}^{\prime} \\
& =\bar{\phi}_{\alpha}\left(-\left(r_{\alpha^{-1}}\right)^{-1}\right) y_{\alpha^{-1}}^{\prime} \\
& =\bar{\phi}_{\alpha}\left(-\left(r_{\alpha^{-1}}\right)^{-1} y_{\alpha^{-1}}\right) \\
& =\bar{\phi}_{\alpha} S_{\alpha}\left(y_{\alpha}\right) .
\end{aligned}
$$

Similarly for $\phi_{\alpha}^{-1} S_{\alpha}^{\prime}\left(y_{\alpha}^{\prime}\right)=S_{\alpha} \phi_{\alpha}^{-1}\left(y_{\alpha}^{\prime}\right)$.

Finally, it is easily see that

$$
\bar{\phi}_{\alpha}\left(A_{\alpha}\left(\chi, r_{\alpha}, \delta_{\alpha}\right)\right) \subseteq A_{\alpha}^{\prime}\left(\chi^{\prime}, r_{\alpha}^{\prime}, \delta_{\alpha}^{\prime}\right)
$$

and

$$
\phi_{\alpha \beta}^{-1}\left(A_{\alpha}^{\prime}\left(\chi^{\prime}, r_{\alpha}^{\prime}, \delta_{\alpha}^{\prime}\right)\right) \subseteq \bar{\phi}_{\alpha}\left(A_{\alpha}\left(\chi, r_{\alpha}, \delta_{\alpha}\right)\right) .
$$

So we conclude that $R$ is isomorphism to $R^{\prime}$ as a Hopf group coalgebra-Ore extension.

Acknowledgments. This work was supported by the National Natural Science Foundation of China (No. 11261063 and 11171183), the Shandong Provincial Natural Science Foundation of China (No. ZR2011AM013).

\section{References}

[1] M. Beattie, S. Dascalescu, and L. Grunenfelder, Constructing pointed Hopf algebras by Ore extensions, J. Algebra 225 (2000), no. 2, 743-770.

[2] M. Beattie, S. Dascalescu, L. Grunenfelder, and C. Nastasescu, Fitness conditions, coFrobenius Hopf algebras and quantum group, J. Algebra 200 (1998), no. 1, 312-333.

[3] A. S. Hegazi, W. Morsi, and M. Mansour, Differential calculus on Hopf group coalgebras, Note Mat. 29 (2009), no. 1, 1-40.

[4] S. Montgomery, Hopf algebras and their actions on rings, CBMS Regional Conference Series in Mathematics, 82, Providence, RI, 1993.

[5] A. Nenciu, Quasitriangular structures for a class of pointed Hopf algebras constructed by Ore extensions, Comm. Algebra 29 (2001), no. 8, 3419-3432.

[6] A. N. Panov, Ore extensions of Hopf algebras, Math. Notes 74 (2003), no. 3-4, 401-410.

[7] M. E. Sweedler, Hopf Algebras, Math. Lecture Notes Ser., Benjamin, New York, 1969.

[8] V. G. Turaev, Homotopy field theory in dimension 3 and crossed group-categories, Preprint GT/0005291,(2000).

[9] — Crossed group-categories, Arab. J. Sci. Eng. Sect. C Theme Issues 33 (2008), no. $2,483-503$. 
[10] A. Van Daele, Multiplier Hopf algebras, Trans. Amer. Math. Soc. 342 (1994), no. 2, 917-932.

[11] A. Virelizier, Hopf group coalgebras, J. Pure Appl. Algebra 171 (2002), no. 1, 75-122.

[12] S. H. Wang, Turaev group coalgebras and twisted Drinfeld double, Indiana Univ. Math. J. 58 (2009), no. 3, 1395-1417.

[13] L. Zhao and D. Lu, Ore Extension of Multiplier Hopf algebras, Comm. Algebra 40 (2012), no. 1, 248-272.

[14] M. Zunino, Double construction for crossed Hopf coalgebra, J. Algebra. 278 (2004), no. $1,43-75$.

[15] — Yetter-Drinfeld modules for crossed structures, J. Pure Appl. Algebra 193 (2004), no. 1-3, 313-343.

DingGuO WANG

School of Mathematical Sciences

Qufu Normal University

Qufu, Shandong 273165, P. R. China

E-mail address: dingguo95@126.com, dgwang@mail.qfnu.edu.cn

DAOWEI Lu

School of Mathematical Sciences

Qufu Normal University

Qufu, Shandong 273165, P. R. China

E-mail address: ludaowei620@sina.com 